\title{
Cross-reaction of Sera from COVID-19 Patients with SARS-CoV Assays
}

\section{Dear Editor,}

SARS-CoV-2 is a new zoonotic coronavirus $(\mathrm{CoV})$ that emerged in Wuhan, China, which was first reported to the World Health Organization (WHO) on 31 December 2019. This coronavirus disease (COVID-19) causes mild to moderate respiratory illness in the majority of patients, but can cause serious complications in the elderly and in those with comorbidities. Poorer resourced countries may not have the capability to equip themselves with complex molecular diagnostic setups. Thus, alternative diagnostic methods maybe crucial to contain outbreaks.

The SARS-CoV-2 is a SARS-related virus with $74.5 \%$ genome identity to SARS-CoV. ${ }^{1}$ The similarities between these 2 viruses were described comprehensively in a recently published article by $\mathrm{Xu}$ et $\mathrm{al}^{2}{ }^{2}$ For structural proteins, including the nucleocapsid $(\mathrm{N})$, matrix $(\mathrm{M})$, and envelope (E), high within-group conservation was maintained, with more modest similarities seen across the entire $\mathrm{CoV}$ family. In contrast, the accessory proteins that distinguish $\mathrm{CoV}$ infections from each other have high variability across the family, and allow viruses to adapt to current and novel hosts. ${ }^{3}$ In a study which described the differences in amino acid substitutions of different proteins for SARS-CoV-2 compared to SARS$\mathrm{CoV}$, it was found that there were no substitutions that occurred in nonstructural protein 7 (nsp7), nsp13, envelope, matrix and accessory proteins $\mathrm{p} 6$ and $8 \mathrm{~b} .{ }^{4}$ The $\mathrm{N}$ protein for SARS-CoV-2 has approximately $90 \%$ similar amino acid identity to the SARS-CoV $\mathrm{N}$ protein and hence the SARS-CoV-2 antibodies against the $\mathrm{N}$ protein would likely recognise and bind the SARS-CoV N protein as well. ${ }^{5}$ Furthermore, a study by Zhou et al showed that the SARS-CoV-2 could be cross-neutralised by horse anti-SARS-CoV serum at a dilution 1:80, confirming the relationship of the 2 viruses. ${ }^{6}$

Based on this knowledge, we postulated that the antibodies produced by COVID-19 patients should result in cross-reactivity to the SARS-CoV total antibody ELISA and indirect immunofluorescence assay (IIFA) tests, which utilise whole SARS-CoV infected cells as the antigen substrate.

Patients' consent and approval from the Ethics Committee were not required for the evaluation of this assay as patient identifiers were removed from all samples.

\section{Methods}

We identified SARS-CoV-2 positive cases which were confirmed by molecular testing of respiratory specimens by real-time RT-PCR, according to the published protocol by Corman et al. ${ }^{7}$ We retrieved residual samples left over from biochemical tests to obtain serial sera for these patients. The Biochemistry Department removed all patient identifiers and assigned random numbers to each patient. Also included were the number of days after onset of illness for each of the retrieved specimens, based on the information obtained from the Infectious Diseases team. Onset of illness was defined as any upper respiratory tract symptoms or fever. For negative controls, 10 samples which were sent for unrelated virology tests from 2 different groups of patients were randomly selected. The first group consisted of 5 sera collected 5 years ago from our archive. The other group comprised 5 sera from patients who were tested negative on two occasions for SARS-CoV-2 by real-time RT-PCR, as part of the enhanced surveillance for patients who presented with pneumonia but did not fulfil the criteria of suspected SARS-CoV-2 infection during this outbreak period.

We performed 2 serological test methods on the selected samples using the SARS-CoV total antibody ELISA test as described by Ksiazek et $\mathrm{al}^{8}$ and Anti-SARS CoV Indirect Immunofluorescence test (IIFT) (IgM and IgG) by Euroimmun (Germany), according to the specified protocols and manufacturer's 
instructions, respectively. Both these tests had previously been validated by the authors and the manufacturer, respectively, to have no cross reactivity with antibodies from other known human coronaviruses.

\section{Results}

There were a total of 7 patients with confirmed COVID-19 admitted to our institution during the study period. A total of 26 samples were retrieved from the Biochemistry Department. The number of samples obtained from each patient ranged from 1 to 9 (mean 3.7), with the earliest taken 1 day after the onset of symptoms and latest at day 24. Five specimens were excluded owing to the narrow interval between samples or close proximity to the date of onset of illness. Figure 1 summarises all the test results for these patients (P1 to P7). Six out of the 7 patients had at least 1 positive antibody result, and seroconversion was demonstrated in 4 patients. The test results were negative for all the negative control samples except for an IgM IIFT result which was deemed indeterminate due to non-specific fluorescence.

\section{Discussion}

In SARS-CoV, both the IgM and IgG antibodies can appear as early as 1 week after diagnosis in more than half of the patients, with the IgM diminishing from week 5 to undetectable levels by week $11 .{ }^{9}$ Other studies found that $80 \%$ of SARS-CoV patients were antibody positive by 8 to 14 days after falling ill, and the mean time to seroconversion was 20 days, with $93 \%$ sensitivity of IgG detection by day $28 .^{10,11}$ A study by Zhang et al, which used an in-house IgM and IgG ELISA test, found that $50 \%$ of their patients were positive for IgM from samples taken on day 0 of hospital admission, which increased to $81 \%$ by day 5 , whereas positive $\operatorname{IgG}$ rates on those same samples increased from $81 \%$ to $100 \%$ in the same period. ${ }^{12}$ However, these rates were based on the number of days from the time of hospital admission rather than from the onset of clinical symptoms. Hence, the early high proportion of positive antibody results reported in this study is not representative of how soon the $\operatorname{IgM}$ and IgG appear after infection. In our current COVID-19 cohort (where sera were available for analysis), $25 \%(1 / 4)$ of the patients had detectable antibodies in the first week of illness, $66.7 \%(4 / 6)$ by the second week, and $100 \%(5 / 5)$ by the third week of illness. These data are somewhat imprecise owing to the limited numbers of patients. In addition, we did not have samples for analysis from P3 in weeks 2 and 3 (we had weeks 1 and 4 only) or from P5 beyond the second week.

Our evaluation study of both the ELISA and IIFA tests on SARS-CoV patients in 2003 showed that overall, the IIFA test was $28.9 \%$ more sensitive than the ELISA test, which explains the results of day 24 for P3. Although the overall results for the IIFA test may be more sensitive, the IIFA IgM test in the COVID-19 patients was found to be less useful in the detection of acute phase of the illness. This is consistent with the findings of a study in SARS-CoV patients where a less frequent (43\%) and robust (less discriminatory) IgM response was found. ${ }^{13}$ However, this cannot be generalised as different assays will have different performances depending on the type of antigen utilised.

There is a possibility that positive antibodies from these tests could be a result of previous exposure to SARS 17 years ago. However, given that only 8,096 cases were reported worldwide and that the virus is not known to still be circulating in the community after it was declared to be contained, with no further reported cases in 2004 , by WHO, this probability seems very small and can be excluded by specific history taking.

The limitations of this study include the relatively small number of patients, inconsistent series of sera and no correlation with clinical severity. Ideally, sera should have been collected at a predetermined regular time interval to determine when $\operatorname{IgM}$ and $\operatorname{IgG}$ can be detected by these assays after infection in COVID-19. Follow up samples 3 to 6 months following infection would also be useful. We also did not take into account other factors which could cause the delay in development of antibodies such as immunosuppressive conditions and other treatment modalities that could affect this. However, this study has provided evidence that antibodies to SARS-Cov-2 cross react to give positive results in existing SARS-CoV assays owing to the similar structural proteins that it shares with SARS-CoV. The positive predictive value of a serological test depends on the prevalence of the virus and thus, in the current situation where there is a recognised outbreak, patients who present with recent compatible symptoms and test positive by these tests are likely to have had exposure to SARS-CoV-2. Compared to molecular methods, serological assays 


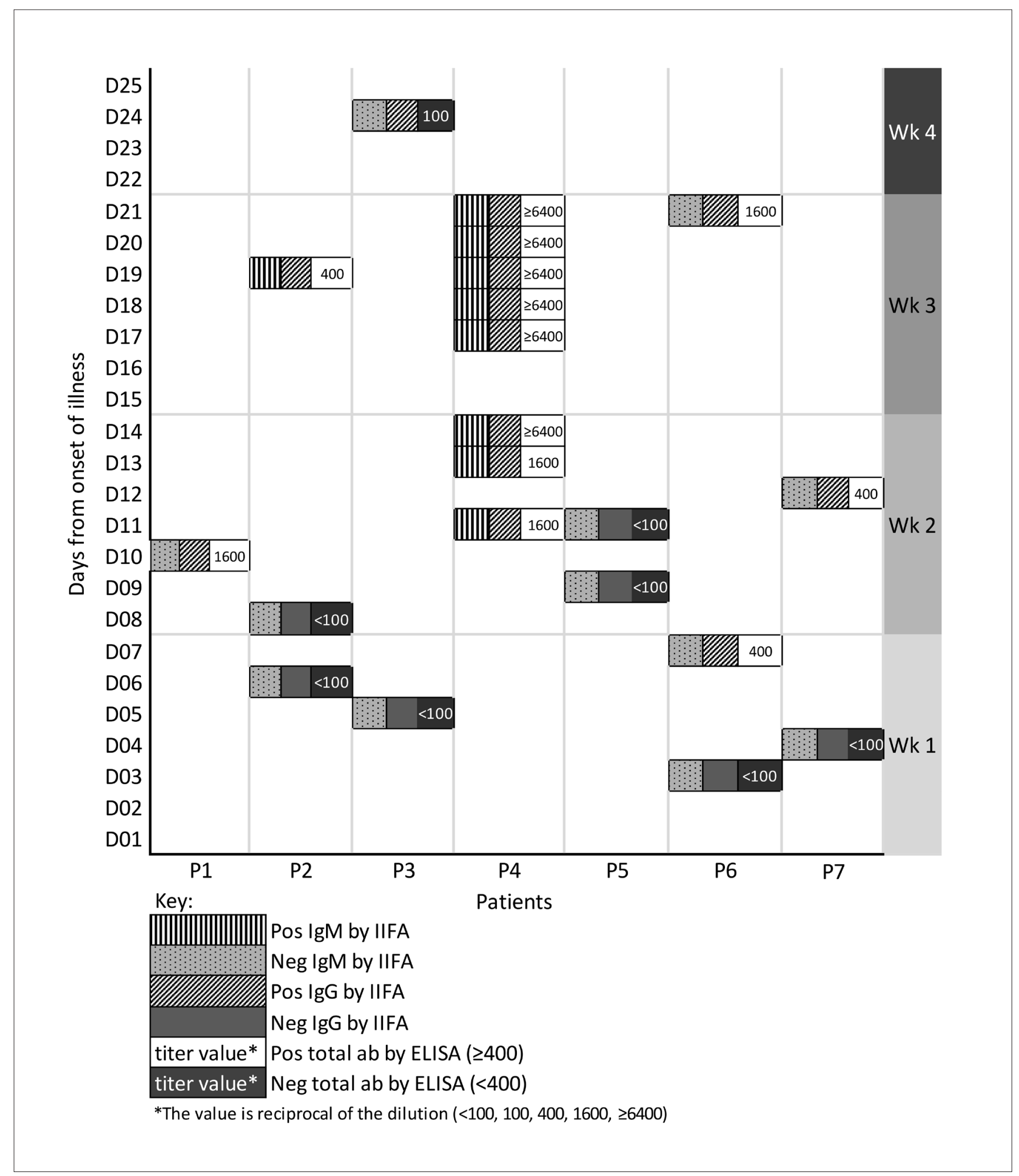

Fig 1. Test Results for the COVID-19 Patients 
have the advantages of lower set-up costs, capacity for large volume processing, shorter turnaround times, are less prone to specimen sampling quality issues, require lower specific technical skills, ${ }^{14}$ have no risk of specimen contamination, involve handling of lower biohazard risk specimens and expose healthcare workers to lower risks during sampling from patients.

In conclusion, we provided proof of concept that the available SARS-CoV antibody assays can reliably detect antibodies in patients with COVID-19 which could be used in this current outbreak situation for serosurveys and as a diagnostic tool for under resourced countries. Further studies would be required to confirm their utility and better determine the time frame when IgM and IgG are detectable in patients exposed to SARS-CoV-2.

\section{Acknowledgments}

We would like to thank the Biochemistry Department in our healthcare institution for its help in retrieving and blinding of specimens, and the Infectious Disease team for providing the onset dates of illness, which were pertinent for the meaningful interpretation of results.

\section{REFERENCES}

1. Huang C, Wang Y, Li X, Ren L, Zhao J, Hu Y, et al. Clinical features of patients infected with 2019 novel coronavirus in Wuhan, China. Lancet. 2020;395:497-506.

2. Xu J, Zhao S, Teng T, Abdalla AE, Zhu W, Xie L, et al. Systematic comparison of two animal-to-human transmitted human coronaviruses: SARS-CoV-2 and SARS-CoV. Viruses, 2020;12,244.

3. Menachery VD; Graham RL; Baric RS. Jumping species-Amechanism for Coronavirus persistence and survival. Curr Opin Virol. 2017;23:1-7.

4. Wu A, Peng Y, Huang B, Ding X, Wang X, Niu P, et al. Genome composition and divergence of the Novel Coronavirus (2019-nCov) originating in China. Cell Host and Microbe. 2020. DOI:10.1016//j. chom.2020.02.001.

5. Gralinski LE, Menachery VD. Return of the Coronavirus: 2019-nCoV. Viruses. 2020;12:135.

6. Zhou P, Yang XL, Wang XG, Ju B, Zhang L, Zhang W, et al. Discovery of a novel coronavirus associated with the recent pneumonia outbreak in humans and its potential bat origin. BioRxiv preprint. DOI: $10.1101 / 2020.01 .22 .914952$.

7. Cormon VM, Landt O, Kaiser M, Molenkamp R, Meijer A, Chu DKW, et al. Detection of 2019 novel coronavirus (2019-nCoV) by real-time RT-PCR. Euro Surveill. 2020;25(3):2000045.

8. Ksiazek TG, Erdman D, Goldsmith CS, Zaki SR, Peret T, Emery S, et al. A novel Coronavirus associated with Severe Acute Respiratory Syndrome. N. Eng. J. Med. 2003;34:1953-1966.

9. Chen W, Xu Z, Mu J, Yang L, Gan H, Mu F, et al. Antibody response and viraemia during the course of severe acute respiratory syndrome (SARS) - associated coronavirus infection. J Med Micro. 2004;53:435-438.

10. Liu X, Shi Y, Li P, Li L, Yi Y, Ma Q, et al. Profile of antibodies to nucleocapsid protein of the Severe Acute Respiratory Syndrome (SARS)-Associated Coronavirus in probable SARS patients. Clin Diag Lab Immunol. Jan 2004;11(1):227-228.

11. Peiris JSM, Chu CM, Cheng VCC, Chan KS, Hung IFN, Poon LLM, et al. Clinical progression and viral load in a community outbreak of coronavirus-associated SARS pneumonia: a prospective study. Lancet. 2003;261:1767-72.

12. Zhang W, Du RH, Li B, Zheng XS, Yang XL, Hu B, et al. Molecular and serological investigation of 2019-nCoV infected patients: implication of multiple shedding routes. Emerg Microbes Infect. 2020;9:1:386-389.

13. Leung DTM, Tam FCH, Ma CH, Chan PKS, Cheung JLK, Niu H, et al. Antibody response of patients with Severe Acute Respiratory Syndrome (SARS) targets the viral nucleocapsid. J. of Inf Dis. 2004;190:379-86.

14. Xiao SY, Wu Y, Liu H. Evolving status of the 2019 novel coronavirus infection: Proposal of conventional serological assays for disease diagnosis and infection monitoring. J Med Viro. 2020;1-4. DOI: $10.1002 /$ jmv. 25702 .

Wei Yee Wan, ${ }^{1}$ FRCPath, Siew Hoon Lim, ${ }^{1} M L Q E$, Eng Hong Seng, ${ }^{1} D C H E$

${ }^{1}$ Department of Microbiology, Singapore General Hospital, Singapore.

Address for Correspondence: Dr Wan Wei Yee, Microbiology Department, Singapore General Hospital, Academia Level 7, 20 College Road, 169608 Singapore.

E-mail: wan.wei.yee@singhealth.com.sg 